\title{
A neotype nomogram based on accessible clinical indicators to predict the overall survival of esophageal cancer patients underwent radical resection
}

\section{Xinye Li ( $\sim$ lxyzhjhz@zju.edu.cn )}

Zhejiang University School of Medicine First Affiliated Hospital https://orcid.org/0000-0001-8527-2256 Jinming Xu

Department of Thoracic Surgery, The First Affiliated Hospital, School of Medicine, Zhejiang University Linhai Zhu

Department of Thoracic Surgery, The First Affiliated Hospital, School of Medicine, Zhejiang University, 79 Qingchun Road, Hangzhou, 310003, China.

\section{Sijia Yang}

Department of Thoracic Surgery, The First Affiliated Hospital, School of Medicine, Zhejiang University, 79 Qingchun Road, Hangzhou, 310003, China.

\section{Li Yu}

Department of Thoracic Surgery, The First Affiliated Hospital, School of Medicine, Zhejiang University, 79 Qingchun Road, Hangzhou, 310003, China.

\section{Wang LV}

Department of Thoracic Surgery, The First Affiliated Hospital, School of Medicine, Zhejiang University, 79 Qingchun Road, Hangzhou, 310003, China.

Jian Hu

Department of Thoracic Surgery, The First Affiliated Hospital, School of Medicine, Zhejiang University, 79 Qingchun Road, Hangzhou, 310003, China.

\section{Research}

Keywords: Esophageal cancer, radical resection, prognosis, AJCC, clinical indicator, nomogram, risk classification

Posted Date: October 9th, 2020

DOI: https://doi.org/10.21203/rs.3.rs-87555/v1

License: (c) (1) This work is licensed under a Creative Commons Attribution 4.0 International License. 
Loading [MathJax]/jax/output/CommonHTML/jax.js

Page 2/25 


\section{Abstract}

Background: Esophageal cancer (EC) is a malignant tumor with dreadful mortality, nomogram is a prognosis tool of great significance in therapeutic guidance and assessment. We aimed to establish a newly-built nomogram for OS prediction of EC patients with radical esophagectomy.

Methods: A total of 311 EC patients underwent radical esophagectomy were retrospectively investigated with their survival and demographic and clinicopathological data. Patients were randomly divided into the primary and validation cohorts. The establishment of nomogram was based on Cox hazard regression analysis in primary cohort, the calibration curves and Harrell's concordance index were performed to verify the predictive accuracy while ROC curves was adopted to reflect the efficacy of nomogram. Kaplan-Meier curves showed the clinical significance of risk classification system and Pearson correlation test was utilized to show the correlation between risk classification system and TNM staging.

Results: The median OS and 5-year survival rate are 44 months and $29.8 \%$ in primary cohort. In validation cohort, they are 52 months and $27.1 \%$, respectively. In Cox hazard regression analysis, we extracted six independent prognostic factors-age, gender, AGR, PRL, N stage, PNI-to establish the nomogram. The Cindex of nomogram is 0.75 in primary cohort and 0.70 in validation cohort. Calibration curves indicated high consistency between accurate and predicted OS in both primary and validation cohorts. ROC curves showed a better efficacy of nomogram compared with AJCC T and N stage. The area under curve (AUC) of primary cohort is 0.801 and 0.727 in validation cohort. Patients in primary cohort were divided into three risk groups according to the nomogram score, the median OS between each group was significantly different. Analogical results were obtained in validation cohort. Furthermore, the risk classification system was strongly correlated to AJCC TNM staging system in total cohort $\left(\mathrm{r}^{2}=0.647, \mathrm{P}<0.001\right)$, and it also demonstrated a better OS prediction efficacy (AUC=0.742).

Conclusions: We established a neotype nomogram and a relevant risk classification system with verified accuracy and efficacy in OS prediction of EC patients after radical esophagectomy. They may provide feasible value in prognosis assessment and treatment guidance prospectively.

\section{Background}

As a malignancy with ascending morbidity and mortality worldwide, esophageal cancer (EC) seriously threatens human life and health.(1) Additionally, as a result of diet and living habits, esophageal cancer ranks as the 6th incidence of malignancy, and the mortality rate of which ranks 4th in China.(2) The most common pathological type of esophageal cancer is squamous cell carcinoma in China while in western nations, it is adenocarcinoma. Radical resection is the major therapy for esophageal cancer. However, due to lack of accurate early diagnosis approaches and effective prognostic indicators, the 5-year overall survival (OS) rate of EC is around $30 \% .{ }^{(3)}$ Recently with the evolution of neoadjuvant chemoradiotherapy, the proanosis of radical esophadeal cancer surgery has been markedly improved.(4) Particularly, an Loading [MathJax]/jax/output/CommonHTML/jax.js 
accurate evaluation of EC patient prognosis contributes to formulating more precise and individualized treatment strategies to prolong survival.

Various factors have a crucial influence on the prognosis of EC patients especially the survival rate which described as independent prognostic factor. With decades of widespread multi-center clinical research, tumor-node-metastasis staging system represented by American Joint Committee on Cancer (AJCC TNM) as well as age, pathological type, treatment patterns are approved as conventional independent prognostic indicators for EC.(5) However, due to the confounding factors that affect the prognosis of EC, single-handed AJCC staging cannot predict the prognosis satisfactorily, especially for patients with the similar staging. $(6,7)$

Nomograms are widely utilized in cancer prognosis for their ability to transform statistical prediction results into a comprehensive quantitative and visual estimate of the probability of an event, such as death or recurrence. $(8,9)$ As a malignancy with poor prognosis and survival, nomogram studies related to EC survival and recurrence are of gigantic significance. The establishment of nomograms integrating conventional factors for EC has been noted in a few studies.(10-12) Furthermore, a series of indicators that can be converted by routine clinical examination parameters have been found to show considerable prognostic potential in EC patients such as albumin to globulin ratio (AGR), neutrophil to lymphocyte ratio (NLR), platelet to lymphocyte ratio (PLR), Prognostic Nutritional Index (PNI), Low-density lipoprotein (LDL).(13-16) However, few studies on the nomogram combining these certified novel prognostic factors in EC are published.

In this study, we aim to retrospectively investigate the independent prognostic factors for OS in EC patients underwent radical esophagectomy and establish an applicable nomogram for internal and external validation and exploring its prediction efficacy compared with AJCC staging.

\section{Materials And Methods}

\subsection{Patients}

The clinicopathological and survival data of 311 patients underwent radical resection of esophageal cancer during 2008 and 2013 in The First Affiliated Hospital of Zhejiang University School of Medicine were retrospectively collected and analyzed. The following are the inclusion criteria: (1) patients with a confirmed pathological diagnosis of EC (squamous or adenous) in first admission; (2) patients without any anti-tumor therapy before surgical esophagectomy; (3) the data of patients are completed. The exclusion criteria followed: (1) patients with a pathology type other than adenocarcinoma or squamous cell carcinoma; (2) surgery not reaching R0 excision; (3) patients with secondary carcinomas assessed by clinical history, imageological examination, or routine laboratory tests; (4) patients with severe and fatal complications after surgery. This study was conducted in accordance with the principles of the Declaration of Helsinki and approved by the Clinical Research Ethics Committee of the First Affiliated Hospital of Zhejiang University School of Medicine.(17) 
The variables of each patients included demographic data: age, gender, smoke and alcohol status, BMl; clinicopathological data: CEA ( $\mathrm{ng} / \mathrm{ml})$, tumor location, differentiated degree, T and $\mathrm{N}$ stage, LDL ( $\mathrm{mmol} / \mathrm{L}$ ); available prognostic indicator: AGR, NLR, PLR, PRL, PNI. All baseline data were obtained in a week before radical esophagectomy.

A. BMI (Body mass index) $=$ Body weight $(\mathrm{kg}) /\left(\right.$ Height $(\mathrm{m})^{\wedge}$ ^2).

B. T and N stage: According to the 7th edition AJCC TNM staging guideline of EC.

C. AGR: Albumin to globulin ratio.

D. NLR: Neutrophil to lymphocyte ratio.

E. PLR: Platelet to lymphocyte ratio.

F. PRL: Positive lymph nodes ratio.

G. LDL: Low-density lipoprotein.

H. PNI: Prognostic Nutritional Index, calculated as $10 \times$ serum albumin $(\mathrm{g} / \mathrm{dL})+0.005 \times$ lymphocyte count (per mm3).(18)

\subsection{Study design and outcome definition}

All included patients are randomly allocated into a primary cohort $(215$ of $311,69.1 \%)$ and a validation cohort (96 of $312,30.9 \%)$. The patients were followed up via clinic visits or telephone interviews, and the overall survival (OS) of the patients were recorded for principle endpoint. The OS was calculated from the time of radical esophagectomy until the time of death with any reasons or until the last follow-up. Study design is described as a flow chart in Fig. 1.

\subsection{Statistical Methods}

Categorical variables were classified based on clinical findings, and continuous variables were transformed into categorical variables based on cut-off points determined by median values or recognized clinical findings. In the balance control between primary and validation cohorts, chi-square was utilized to show the difference of proportion in each variable.

In primary cohort, univariable and multivariable Cox's proportional hazards regression for OS was performed, and variables with $P$ values less than 0.05 in the multivariable Cox's proportional hazards regression were utilized to establish a predictive nomogram model.(8)

The predictive accuracy of the nomogram model was performed using the Harrell's concordance index (Cindex) and calibration curves, the larger value of C-index indicated more efficient ability to correctly discriminate the prediction of outcome.(19) Calibration curves for 1-year, 3-year, 5-year OS were performed using a bootstrap method to quantify the modeling strategy of nomogram. Receiving operative characteristics (ROC) curves were used to compare the prediction efficacy of nomogram with AJCC staging. All the methods above were equally implemented in validation cohorts for external verification. 
Furthermore, based on the total score from the predictive nomogram model, patients were divided into 3 risk groups (low risk, intermediate risk, high risk) in both primary and validation cohorts. Kaplan-Meier curves and the log-rank test were used to illustrate and compare the OS of patients in the different risk groups.

In the total cohort, chi-square and Pearson correlation test were applied to show the correlation between risk classification system and AJCC TNM staging system.

All the statistical analyses and graphics above were performed using the SPSS 25.0 statistical package (SPSS Inc., Chicago, IL, USA) and R version 3.6.1 (R Foundation for Statistical Computing, Vienna, Austria) and GraphPad Prism 8.2.1 (GraphPad Software, La Jolla, CA). P values less than 0.05 in twosided test were considered statistically significant.

\section{Results}

\subsection{Characteristics of patients in randomized primary and validation cohorts}

A total of 311 eligible patients who underwent radical esophagectomy in First Affiliated Hospital of Zhejiang University School of Medicine were enrolled and randomly grouped into primary and validation cohorts. The median age is 63 (range $40-83, S D=7.76$ ) years old in all patients. Moreover, the median OS and 5-year survival rate are 44 months and $29.8 \%$ in primary cohort. In validation cohort, they are 52 months and $27.1 \%$, respectively. The continuous variables, including BMI, NLR, PLR, AGR, LDL, CEA, $\mathrm{PNI}, \mathrm{PRL}$, are transformed into binary categorical variables according to cut-off values determined by median or clinically recognized results. All patients' baseline demographic and clinicopathological characteristics are balanced in primary and validation cohorts displayed in Table 1. 
Table 1

Baseline demographic and clinicopathological characteristics in primary and validation cohorts

\begin{tabular}{|c|c|c|c|}
\hline & $\begin{array}{l}\text { Primary cohort } \\
(\mathrm{N}=215)\end{array}$ & $\begin{array}{l}\text { Validation cohort } \\
(\mathrm{N}=96)\end{array}$ & $P$ value ${ }^{a}$ \\
\hline Characteristics & No. (\%) & No. (\%) & - \\
\hline \multicolumn{4}{|l|}{ Age (years) } \\
\hline$\geq 65$ & $87(40.5)$ & $36(37.5)$ & 0.621 \\
\hline$<65$ & $128(59.5)$ & $60(62.5)$ & \\
\hline \multicolumn{4}{|l|}{ Gender } \\
\hline Male & $190(88.4)$ & $82(87.5)$ & 0.467 \\
\hline Female & $25(11.6)$ & $14(12.5)$ & \\
\hline \multicolumn{4}{|l|}{ Smoke status } \\
\hline Yes & $144(67.0)$ & $69(71.9)$ & 0.390 \\
\hline No & $71(33.0)$ & $27(28.1)$ & \\
\hline \multicolumn{4}{|l|}{ Alcohol status } \\
\hline Yes & $136(63.3)$ & $62(64.6)$ & 0.822 \\
\hline No & 79 (36.7) & $34(35.4)$ & \\
\hline \multicolumn{4}{|l|}{ BMI } \\
\hline$\geq 22.13$ & $116(54.0)$ & $59(61.5)$ & 0.218 \\
\hline$<22.13$ & $99(46.0)$ & $37(38.5)$ & \\
\hline \multicolumn{4}{|l|}{ NLR } \\
\hline$\geq 2.22$ & $106(49.3)$ & $48(50)$ & 0.909 \\
\hline$<2.22$ & $109(50.7)$ & $48(50)$ & \\
\hline \multicolumn{4}{|l|}{ PLR } \\
\hline$\geq 123.51$ & $105(48.8)$ & $49(51.0)$ & 0.719 \\
\hline$<123.51$ & $110(51.2)$ & $47(49.0)$ & \\
\hline
\end{tabular}

BMI: Body mass index, AGR: Albumin to globulin ratio, NLR: Neutrophil to lymphocyte ratio, PLR: Platelet to lymphocyte ratio, PRL: Positive lymph nodes ratio, LDL: Low-density lipoprotein, PNI: Prognostic Nutritional Index. 


\begin{tabular}{|c|c|c|c|}
\hline & $\begin{array}{l}\text { Primary cohort } \\
(\mathrm{N}=215)\end{array}$ & $\begin{array}{l}\text { Validation cohort } \\
(\mathrm{N}=96)\end{array}$ & $P$ value ${ }^{\text {a }}$ \\
\hline$\geq 1.61$ & $112(52.1)$ & $44(45.8)$ & 0.308 \\
\hline$<1.61$ & 103 (47.9) & $52(54.2)$ & \\
\hline \multicolumn{4}{|l|}{ LDL (mmol/L) } \\
\hline$\geq 2.42$ & $111(51.6)$ & $50(52.1)$ & 0.941 \\
\hline$<2.42$ & $104(48.4)$ & $46(47.9)$ & \\
\hline \multicolumn{4}{|l|}{ CEA (ng/ml) } \\
\hline$\geq 5$ & $27(12.6)$ & $13(13.5)$ & 0.811 \\
\hline$<5$ & 188 (87.4) & $83(86.5)$ & \\
\hline \multicolumn{4}{|l|}{ PNI } \\
\hline$\geq 50$ & 109 (50.7) & 45 (46.9) & 0.533 \\
\hline$<50$ & 106 (49.3) & $51(53.1)$ & \\
\hline \multicolumn{4}{|l|}{ Tumor location } \\
\hline Upper & $14(7.0)$ & $5(5.2)$ & 0.658 \\
\hline Middle or lower & 201 (93.0) & $91(94.8)$ & \\
\hline \multicolumn{4}{|l|}{ Differentiation } \\
\hline Poor & $24(11.2)$ & 7 (7.3) & 0.182 \\
\hline Moderate & 119 (55.3) & $47(49.0)$ & \\
\hline Well & 72 (33.5) & $42(43.7)$ & \\
\hline \multicolumn{4}{|l|}{ T stage ${ }^{b}$} \\
\hline Tis/l & $39(18.1)$ & $16(16.7)$ & 0.463 \\
\hline II & 36 (16.7) & $10(10.4)$ & \\
\hline III & $122(56.7)$ & $62(64.6)$ & \\
\hline IV & $18(8.4)$ & $8(8.3)$ & \\
\hline \multicolumn{4}{|l|}{ N stage ${ }^{b}$} \\
\hline \multicolumn{4}{|c|}{$\begin{array}{l}\text { BMI: Body mass index, AGR: Albumin to globulin ratio, NLR: Neutrophil to lymphocyte ratio, PLR } \\
\text { Platelet to lymphocyte ratio, PRL: Positive lymph nodes ratio, LDL: Low-density lipoprotein, PNI: } \\
\text { Prognostic Nutritional Index. }\end{array}$} \\
\hline
\end{tabular}




\begin{tabular}{|c|c|c|c|}
\hline & $\begin{array}{l}\text { Primary cohort } \\
(\mathrm{N}=215)\end{array}$ & $\begin{array}{l}\text { Validation cohort } \\
(\mathrm{N}=96)\end{array}$ & $P$ value ${ }^{a}$ \\
\hline $\mathrm{N} 0+\mathrm{N} 1$ & 176 (81.9) & 78 (81.3) & 0.898 \\
\hline $\mathrm{N} 2+\mathrm{N} 3$ & 39 (18.1) & 18 (18.7) & \\
\hline \multicolumn{4}{|l|}{ PRL } \\
\hline$\geq 0.1$ & 68 (31.6) & $29(30.2)$ & 0.803 \\
\hline$<0.1$ & $147(68.4)$ & $67(69.8)$ & \\
\hline \multicolumn{4}{|c|}{$\begin{array}{l}\text { BMI: Body mass index, AGR: Albumin to globulin ratio, NLR: Neutrophil to lymphocyte ratio, PLR } \\
\text { Platelet to lymphocyte ratio, PRL: Positive lymph nodes ratio, LDL: Low-density lipoprotein, PNI: } \\
\text { Prognostic Nutritional Index. }\end{array}$} \\
\hline
\end{tabular}

\subsection{Cox regression analysis and optimization of variables for nomogram in primary cohort}

Univariable and multivariable Cox hazard regression analysis for OS in primary cohort is showed in Table 2. In univariate Cox hazard regression model, variables including age ( $\geq 65 \mathrm{y}$ vs. $<65 \mathrm{y}$ ), gender (Male vs. female), differentiation (Poor vs. moderately vs. well), AJCC T stage, modified N stage (NO-N1 vs. N2-N3), PLR ( $\geq 123.51$ vs. $<123.51$ ), NLR ( $\geq 2.22$ vs. $<2.21$ ), AGR ( $\geq 1.61$ vs. $<1.61)$, CEA ( $\geq 5 \mathrm{ug} / \mathrm{L}$ vs. $<5 \mathrm{ug} / \mathrm{L}), \mathrm{PRL}(\geq 0.1 \mathrm{vs} .<0.1), \mathrm{PNI}(\geq 50 \mathrm{vs} .<50$ ) are significantly related to $0 \mathrm{~S}$ with $\mathrm{P}$ values less than 0.05 . For optimization of variables to establish a nomogram predictive model, a subsequent multivariable Cox hazard regression analysis in the variables above was applied, and six independent factors (age, gender, AGR, N stage, PNI, PRL) for the OS prediction in radical esophagectomy patients were chosen for the following validation of nomogram. 
Table 2

Univariable and multivariable Cox hazard analysis for OS in primary cohort

\section{Univariable analysis}

Age (years)

$\geq 65$ vs. $<65$

Gender

Male vs. female

2.889

$1.346-6.201$

\section{Smoke status}

Yes vs. No

Alcohol status

Yes vs. No

0.855

$0.614-1.275$

\section{Tumor location}

Middle/lower vs. Upper

Differentiation

Well

Moderate

Poor

T stage

Tis/l

II

III

IV

\section{N stage}

N0-N1 vs. N2-N3

\section{BMI}

$\geq 22$ vs. $<22$

0.752

Ref-

NA

$0.822-3.555$

2.689

3.543

3.904

$2.602-5.857$

\section{NLR}

BMI: Body mass index, AGR: Albumin to globulin ratio, NLR: Neutrophil to lymphocyte ratio, PLR: Platelet to lymphocyte ratio, PRL: Positive lymph nodes ratio, LDL: Low-density lipoprotein, PNI: Prognostic Nutritional Index, OS: Overall survival, HR: hazard ratio, Cl: confidence interval.
0.006

0.511

0.151

$<0.001$

$P$ value $^{\mathrm{c}}$

0.014

0.240

0.882

0.026

NA

0.053

0.010

0.001

NA

0.001

0.002

0.118

Loading [MathJax]/jax/output/CommonHTML/jax.js i considered as statistical significance. 


\begin{tabular}{|c|c|c|c|}
\hline Univariable analysis & HR & $95 \% \mathrm{Cl}$ of HR & $P$ value ${ }^{c}$ \\
\hline$\geq 2.22$ vs. $<2.21$ & 1.438 & $1.004-2.060$ & 0.047 \\
\hline \multicolumn{4}{|l|}{ PLR } \\
\hline$\geq 123.51$ vs. $<123.51$ & 1.552 & $1.083-2.225$ & 0.017 \\
\hline \multicolumn{4}{|l|}{ AGR } \\
\hline$\geq 1.61$ vs. $<1.61$ & 0.490 & $0.341-0.705$ & $<0.001$ \\
\hline \multicolumn{4}{|l|}{ LDL (mmol/L) } \\
\hline$\geq 2.42$ vs. $<2.42$ & 0.754 & $0.527-1.079$ & 0.122 \\
\hline \multicolumn{4}{|l|}{ PNI } \\
\hline$\geq 50$ vs. $<50$ & 0.563 & $0.394-0.806$ & 0.002 \\
\hline \multicolumn{4}{|l|}{ CEA (mmol/L) } \\
\hline$\geq 5 \mathrm{ug} / \mathrm{L}$ vs. $<5 \mathrm{ug} / \mathrm{L}$ & 2.038 & $1.259-3.299$ & 0.004 \\
\hline \multicolumn{4}{|l|}{ PRL } \\
\hline$\geq 0.1$ vs. $<0.1$ & 3.974 & $2.758-5.726$ & $<0.001$ \\
\hline Multivariable analysis & $\mathrm{HR}$ & $95 \% \mathrm{Cl}$ of $\mathrm{HR}$ & $P$ value ${ }^{c}$ \\
\hline \multicolumn{4}{|l|}{ Age (years) } \\
\hline$\geq 65$ vs. $<65$ & 1.426 & $0.984-2.065$ & 0.061 \\
\hline \multicolumn{4}{|l|}{ Gender } \\
\hline Male vs. female & 2.417 & $1.123-5.204$ & 0.024 \\
\hline \multicolumn{4}{|l|}{ N stage } \\
\hline N0-N1 vs. N2-N3 & 1.766 & $1.084-2.877$ & 0.022 \\
\hline \multicolumn{4}{|l|}{ AGR } \\
\hline$\geq 1.61$ vs. $<1.61$ & 0.574 & $0.395-0.834$ & 0.004 \\
\hline \multicolumn{4}{|l|}{ PNI } \\
\hline$\geq 50$ vs. $<50$ & 0.676 & $0.463-0.987$ & 0.043 \\
\hline \multicolumn{4}{|l|}{ PRL } \\
\hline \multicolumn{4}{|c|}{$\begin{array}{l}\text { BMI: Body mass index, AGR: Albumin to globulin ratio, NLR: Neutrophil to lymphocyte ratio, PLR: } \\
\text { Platelet to lymphocyte ratio, PRL: Positive lymph nodes ratio, LDL: Low-density lipoprotein, PNI: } \\
\text { Prognostic Nutritional Index, OS: Overall survival, HR: hazard ratio, Cl: confidence interval. }\end{array}$} \\
\hline
\end{tabular}




\begin{tabular}{|lccc|}
\hline Univariable analysis & HR & 95\% Cl of HR & Pvalue $^{\text {c }}$ \\
\hline$\geq 0.1$ vs. $<0.1$ & 2.970 & $1.902-4.638$ & $<0.001$ \\
\hline $\begin{array}{l}\text { BMl: Body mass index, AGR: Albumin to globulin ratio, } \\
\text { Platelet to lymphocyte ratio, PRL: Positive lymph nodes ratio, LDL: Low-density lipoprotein, PNI: } \\
\text { Prognostic Nutritional Index, OS: Overall survival, HR: hazard ratio, Cl: confidence interval. }\end{array}$ \\
\hline C. Cox hazard regression analysis, P< $<0.05$ considered as statistical significance.
\end{tabular}

\subsection{Establishment of a visual nomogram and internal/external validation}

Based on the six independent factors obtained in multivariable Cox hazard regression analysis, a nomogram characterized by scale line and score weight reflected to 1-, 3-, 5-year OS prediction was established in Fig. 2. The concordance index (C-index) and calibration curve were used in primary and validation cohorts for internal/external validation, respectively. The $\mathrm{C}$-index of the nomogram was 0.75 $(95 \% \mathrm{Cl}, 0.68-0.82)$ in primary cohort, and $0.70(95 \% \mathrm{Cl}, 0.65-0.75)$ in validation cohort, which demonstrated an outstanding prediction accuracy of the nomogram. Furthermore, a set of calibration curves demonstrated the consistency between actual OS and nomogram predictive OS (Fig. 3).

\subsection{ROC of nomogram in prediction OS compared with AJCC T stage and N stage}

A total nomogram-related score (TNS) was calculated in each patient according to the summation of score corresponding to the status of variables in nomogram. The receiver operating characteristics curves (ROC) of TNS, conventional 7th AJCC T stage and N stage for OS prediction was plotted in Fig. 4. The area under the curves (AUC) was evidently distinct in TNS $(0.801,95 \% \mathrm{Cl} 0.744-0.859)$ compared with T stage $(0.629,95 \% \mathrm{Cl} 0.552-0.705)$ and $\mathrm{N}$ stage $(0.693,95 \% \mathrm{Cl} 0.623-0.763)$ in primary cohort (Fig. $4 \mathrm{~A})$. In validation cohort, the AUC of TNS is $0.727(95 \% \mathrm{Cl} 0.626-0.829)$ which was distinct to T stage $(0.624$, $95 \% \mathrm{Cl} 0.510-0.739)$ and $\mathrm{N}$ stage $(0.614,95 \% \mathrm{Cl} 0.502-0.726)$ in Fig. 4B. This result manifested that TNS is a better OS prediction capability in patients underwent radical esophagectomy.

\subsection{Nomogram based risk classification system}

In the primary group, all patients were equally divided into high $(71 / 215$, score $\geq 21.8)$, intermediate $(72 / 215,21.8>$ score $\geq 13.2)$ and low risk groups $(72 / 215$, score $<13.2)$ according to the TNS. The KaplanMeier curves analysis showed significant difference in primary cohorts with a median OS of NA, 51 months, 18 months in low, intermediate and high risk groups (Fig. 5A), the HR of intermediate and high risk groups was $3.221(95 \% \mathrm{Cl} 1.933-5.368, \mathrm{P}<0.001)$ and $6.329(95 \% \mathrm{Cl} 3.964-10.11, \mathrm{P}<0.001)$, referring to low risk group. In addition, equivalent risk classification was applied for validation cohort (Fig. 5B), and analogous results were found. The median OS was NA, 44 months, 28 months in low, intermediate and high risk group while the HR of intermediate and high risk group was $2.684(95 \% \mathrm{Cl}$ $1.688-5.188, \mathrm{P}=0.009)$ and $5.721(95 \% \mathrm{Cl} 2.499-13.10, \mathrm{P} \otimes 0.001)$, referring to low risk group (Table 3$)$. 
Table 3

Log-rank test of the risk groups based on the nomogram model in primary and validation cohorts

\begin{tabular}{|c|c|c|c|c|c|c|}
\hline & \multicolumn{2}{|c|}{ Primary cohort } & \multicolumn{4}{|c|}{ Validation cohort } \\
\hline & Low & Inter- & High & Low & Inter- & High \\
\hline No. (\%) & $\begin{array}{l}72 \\
(33.5)\end{array}$ & $72(33.5)$ & $71(33.0)$ & $\begin{array}{l}38 \\
(39.6)\end{array}$ & $41(42.7)$ & $17(17.7)$ \\
\hline $\begin{array}{l}\text { Median OS } \\
\text { (Months) }\end{array}$ & $N A^{d}$ & 51 & 18 & $N A^{d}$ & 44 & 28 \\
\hline $\mathrm{HR}$ & Ref- & 3.221 & 6.329 & Ref- & 2.684 & 5.721 \\
\hline $95 \% \mathrm{Cl}$ of $\mathrm{HR}$ & Ref- & $\begin{array}{l}1.933- \\
5.368\end{array}$ & $\begin{array}{l}3.964- \\
10.11\end{array}$ & Ref- & $\begin{array}{l}1.688- \\
5.188\end{array}$ & $\begin{array}{l}2.499- \\
13.10\end{array}$ \\
\hline$P$ value $\mathrm{e}^{\mathrm{e}}$ & Ref- & $<0.001$ & $<0.001$ & Ref- & 0.009 & $<0.001$ \\
\hline \multicolumn{7}{|c|}{ OS: Overall survival, HR: hazard ratio, Cl: confidence interval, Inter-: Intermediate } \\
\hline $\begin{array}{l}\text { d. Less than } \\
\text { statistical sig }\end{array}$ & $\begin{array}{l}\text { he deat } \\
\text { ce }\end{array}$ & luring the & e follow-u & rank te & $P<0.05 \mathrm{col}$ & lered as \\
\hline
\end{tabular}

\subsection{Comparison of nomo-related risk classification system with AJCC TNM staging}

All patients in total cohort were divided into different risk groups according to the calculated TNS. $R^{\star} \mathrm{C}$ contingency in Table 4 showed the risk classification was strongly correlated to AJCC TNM stages $\left(r^{2}=\right.$ $0.647, \mathrm{P}<0.001$ ). Additionally, Kaplan-Meier curves in Fig. 6A showed significant difference in OS of patients between risk classification and TNM stages (log-rank: $P<0.001$ ), and a larger AUC of risk classification system than TNM stages (0.744 vs. 0.699$)$ was displayed in Fig. $6 \mathrm{~B}$. 
Table 4

$\mathrm{R}^{\star} \mathrm{C}$ contingency tables of all patients with TNS-related risk in correlation with AJCC TNM stage

\begin{tabular}{|c|c|c|c|c|c|c|c|}
\hline & & $\begin{array}{l}\text { Low risk } \\
(\mathrm{N})\end{array}$ & $\begin{array}{l}\text { Inter- } \\
\text { risk(N) }\end{array}$ & $\begin{array}{l}\operatorname{High} \\
\operatorname{risk}(\mathrm{N})\end{array}$ & Total(N) & $\begin{array}{l}P \\
\text { value }^{g}\end{array}$ & Correlation ${ }^{\mathrm{h}}$ \\
\hline \multirow{8}{*}{$\begin{array}{l}\text { TNM } \\
\text { stage }\end{array}$} & $I^{f}$ & 30 & 18 & 0 & 48 & & \\
\hline & IB & 6 & 3 & 0 & 9 & & \\
\hline & IIA & 7 & 12 & 0 & 19 & & \\
\hline & IIB & 49 & 45 & 10 & 104 & $<0.001$ & $r^{2}=0.647$ \\
\hline & IIIA & 10 & 26 & 34 & 70 & & $P<0.001$ \\
\hline & IIIB & 1 & 6 & 24 & 31 & & \\
\hline & IIIC & 0 & 1 & 29 & 30 & & \\
\hline & Total & 103 & 111 & 97 & 311 & & \\
\hline \multicolumn{8}{|c|}{ TNS: Total nomogram-related score, AJCC: American joint Committee on cancer, Inter-: Intermediate. } \\
\hline \multicolumn{8}{|c|}{ f. According to the definition of 7th AJCC TNM staging system. } \\
\hline \multicolumn{8}{|c|}{ g. chi-square test, h. Pearson Correlation analysis, $\mathrm{P}<0.05$ considered as statistical significance } \\
\hline
\end{tabular}

\section{Discussion}

The prognostic factors in EC patients are known to be complicated, thus, an accurate and comprehensive prognostic method is of great significance for the evaluation of esophageal cancer and the optimization of treatment strategies. So far, TNM staging system proposed by AJCC has been widely accepted for the assessment of cancers including EC. For patients who are adapted to surgery, determining explicit $T$ and $\mathrm{N}$ staging is the most crucial task throughout.(20) However, the TNM staging system mainly represents anatomical relevance, sometimes does not fully reflect the prognosis. Therefore, a type of model called nomogram that can incorporate multiple prognostic factors is maturing. Recently, nomograms are popularly investigated in cancer-related survival, recurrence and metastasis with considerable potential. (21-24) Overall survival (OS) is regarded as a most commonly used outcome for cancer-related survival prognosis with confounding factors while the nomogram studies on EC-related OS prediction are less of quantity. We involved some classical and novel factors which are clinically available to establish a nomogram which is unprecedented and innovative.

By means of literature review and clinical experience, a set of factors were included for the initial analysis in EC patients underwent radical esophagectomy. In addition to some conventional demographic and clinicopathological factors, such as aqe, qender, tumor location, tumor differentiation, $\mathrm{T}$ and $\mathrm{N}$ staging, Loading [MathJax]/jax/output/CommonHTML/jax.js 
etc., the verified cancer-related prognostic factors, including Albumin to globulin ratio (AGR), Neutrophil to lymphocyte ratio (NLR), Platelet to lymphocyte ratio (PLR), Positive lymph nodes ratio (PRL), Low-density lipoprotein (LDL), and Prognostic Nutritional Index (PNI) are candidates of nomogram establishment.(25, 26) Among them, six factors including age, gender, $N$ stage (with modified), AGR, PRL, PNI were identified as independent prognostic factors by statistical Cox hazard regression analysis, and the Hazard Ratio (HR) of each factor displays the weight of score in the nomogram.(9)

For demographic characteristics, we found that two factors, age and gender, were associated with OS of EC patients after radical esophagectomy, and thus were included into the establishment of nomogram. As a matter of genetic difference, males have been found with higher incidence and mortality in a variety of malignancies, as found in various studies and confirmed in ours. $(1,27,28)$ In addition to cancerrelated factors, it cannot be ignored in the prognosis of elderly cancer patients that the pathological mechanisms induced by aging may cause more nutritional and metabolic diseases and disorders such as amyotrophy, metabolism damage and neurological disease, contributing to the impediment of longevity.(29-31) In the result of our study, the age over 65 years old was certified as an independent factor for OS prediction, conforming to the interpretation mentioned above.

Further in comparison with some nomogram studies in resectable EC patients, Shao's study focuses on the predictive ability of inflammation-related factors for OS, and accordingly building a nomogram model. His study is partially similar to our study, and some factors, including PLR and NLR, are selected to be used in our initial analysis.(32) However, with no statistical difference found, they were removed from multivariable Cox hazard regression analysis in our study. The above situation indicates that the factors affecting the OS of radical resection EC patients are very complicated. PLR and NLR are not only related to the prognosis of malignant tumors, but also determined in inflammatory diseases such as Rheumatic Disease and cardiovascular diseases. $(33,34) \mathrm{EC}$ is dependent on the complex interaction between the tumor and the hosts' inflammatory response, so the distinction of PLR and NLR shown between Shao's study and ours imply that the independence of factors affecting prognosis are unseparated from the integrity of the individual physiology.(35) As aforementioned, a low AGR is associated with increased cancer mortality in cancer patients, a generally healthy screened population study proves AGR as a risk factor for cancer incidence and mortality, in both short- and long-term cohorts.(36) There is biological plausibility for the link between low AGR and increased cancer incidence and mortality that an increase of cytokines on account of tumor microenvironment may elevate the total protein levels with induced albumin synthesis suppression in the liver. $(37,38)$ As for EC patients, a malnutrition status relevant decreased serum albumin and AGR level could be another mechanism of poor survival. PNI is composed of serum albumin level and lymphocyte count as a calculative index which is widely utilized for prognostic evaluation of various malignancies. As a factor reflects to tumor-related nutritional status and system inflammation response, the role of PNI in EC patients' prognosis is highly desirable for study. Kazuo's study demonstrated the predictive role of PNI in EC patients outcome and its inseparable relationship with Tumor-infiltrating lymphocytes (TILs).(15) As proved, PNI was significantly associated with OS in Cox hazard regression analysis with a negative correlation of HR 0.676 (95\% Cl: $0.463-0.987)$ Loading [MathJax]/jax/output/CommonHTML/jax.js mogram for OS prediction in resectable EC patients. 
Moreover, the laboratory-sourced factors we selected are all preoperative to eliminate the inflammatory and metabolic bias caused by surgery.

For resectable EC patients, though there is a gap in skills between surgeons, the lymph node dissection degree is considered as an effective adjudication, extended lymph node dissection proves a significant amelioration on the prognosis of EC patients. $(39,40)$ Recently, the ratio of positive retrieved lymph nodes to total number of retrieved lymph nodes-known as the positive lymph node ratio (abbreviation as PRL or LNR) -has been shown to be a superior indicator of survival in EC. $(41,42)$ Compared with $N$ stage according to AJCC, it not only reflects the quantity but the extent of the lymphatic metastasis, especially for the EC patients with less than two-field lymph node dissection or underestimated $\mathrm{N}$ staging due to insufficient retrieve of positive lymph nodes.(43) Evidently, PRL showed a higher HR of 2.970 (95\% Cl: $1.902-4.638$ ) and greatest weight in nomogram compared with $\mathrm{N}$ stage in our study.

Furthermore, we used the method of calibration curve and C-index to verify our nomogram model internally and externally with a considerable accuracy and consistency. Additionally, ROC curves of the nomogram were performed for the prediction of OS, and area under curve (AUC) is the crucial index to discriminate the accuracy of different prediction methods. The larger value of the AUC (no less than 0.5) manifests more outstanding predictive ability.(44) Compared with AJCC T and N staging strategy, we observed that the AUC of the nomogram in the primary cohort reached $0.801(95 \% \mathrm{Cl}: 0.744-0.859)$, which was significantly higher than the $T$ and $\mathrm{N}$ stages. Despite the fact that the AUC of nomogram in validation cohort dropped to 0.727 (95\%Cl: 0.626-0.829), it's still superior to AJCC T and N staging in predicting OS. Eventually, by dividing the primary cohort into three equal risk groups according to the total nomogram score (TNS) of each patient, the OS of the three groups showed a significant difference. Similar result was also observed in validation cohort. This indicates that the nomogram model, if wellestablished, can demonstrate favorable clinical applicability for the prediction of OS in EC patients underwent radical esophagectomy.

In the end, we performed analysis based on the risk classification system in total cohort, comparing with an authoritative tumor staging system AJCC TNM staging to identify its comprehensive application clinically. Eventually, the risk classification system showed strong correlation with TNM staging $\left(r^{2}=\right.$ $0.647, P<0.001)$, and a better efficacy in OS prediction than TNM staging was informed by log-rank test and ROC curves.

In contrast with studies on nomogram in EC patients, $\mathrm{PNI}$, which is scarcely investigated in nomogram of EC patients, was integrated into our study, together with internal and external validation of nomogram. Besides, an applicable risk classification system based on nomogram is developed in our study. $(11,16$, 32)

Several limitations exist in our study. Firstly, it's a retrospective analysis with probably inherent bias. Secondly, the primary and validation cohorts of this study are from a single center. Thirdly, the threshold 
index of factors mentioned in our study is heterogeneous. Therefore, a large-sample, multi-center randomized trial needs to be further verified.

\section{Conclusions}

We established and verified a nomogram composed of six clinically accessible factors including a corresponding risk classification system for OS prediction in EC patients underwent radical esophagectomy. The nomogram showed considerable accuracy and efficacy compared with conventional AJCC staging system. The results can be applied to instruct prospective clinical applications, such as patient counseling, prognosis assessment and individualized therapeutic decisionmaking.

\section{Declarations}

\section{Ethics approval and consent to participate}

This study was conducted in accordance with the principles of the Declaration of Helsinki, all participating patients signed an informed consent form and this study was approved by the Clinical Research Ethics Committee of the First Affiliated Hospital of Zhejiang University School of Medicine.

\section{Consent for publication}

Not applicable.

\section{Availability of data and materials}

The datasets used and/or analysed during the current study are available from the corresponding author on reasonable request.

\section{Competing interests}

The authors declare that they have no competing interests.

\section{Funding}

This work is supported by funds from Zhejiang Research Center for lung cancer diagnosis and treatment technology (JBZX-202007), the National key research and development plan (2017YFC0113500), Major science and technology special project of Zhejiang province (2020C03058), Traditional Chinese Medicine (Integrated Traditional Chinese and Western Medicine) key subject of Zhejiang province (2017-XK-A33).

\section{Authors' contributions}

All the authors contributed to the completion of this manuscript, including the design of study (LXY, HJ), callontinn of alininal data (X IM VC I VI) ctatistical analysis (LXY, ZLH), draft writing (LXY, LW), study Loading [MathJax]/jax/output/CommonHTML/jax.js 
guidance and assistance $(\mathrm{HJ})$.

\section{Acknowledgements}

We thank to the support for study provided by the First Affiliated Hospital of Zhejiang University School of Medicine and all the authors sincerely appreciate to the editorial team and reviewers for their profound advice.

\section{References}

1. Siegel RL, Miller KD, Jemal A. Cancer statistics, 2018. CA Cancer J Clin. 2018;68(1):7-30.

2. Chen W, Zheng R, Baade PD, Zhang S, Zeng H, Bray F, et al. Cancer statistics in China, 2015. CA Cancer J Clin. 2016;66(2):115-32.

3. Pennathur A, Gibson MK, Jobe BA, Luketich JD. Oesophageal carcinoma. Lancet. 2013;381(9864):400-12.

4. Shapiro J, van Lanschot JJB, Hulshof M, van Hagen P, van Berge Henegouwen MI, Wijnhoven BPL, et al. Neoadjuvant chemoradiotherapy plus surgery versus surgery alone for oesophageal or junctional cancer (CROSS): long-term results of a randomised controlled trial. Lancet Oncol. 2015;16(9):1090-8.

5. Hsu PK, Chen HS, Liu CC, Wu SC. Application of the Eighth AJCC TNM Staging System in Patients With Esophageal Squamous Cell Carcinoma. Ann Thorac Surg. 2018;105(5):1516-22.

6. Reeh M, Nentwich MF, von Loga K, Schade J, Uzunoglu FG, Koenig AM, et al. An attempt at validation of the Seventh edition of the classification by the International Union Against Cancer for esophageal carcinoma. Ann Thorac Surg. 2012;93(3):890-6.

7. Shao Y, Geng Y, Gu W, Ning Z, Huang J, Pei H, et al. Assessment of Lymph Node Ratio to Replace the pN Categories System of Classification of the TNM System in Esophageal Squamous Cell Carcinoma. J Thorac Oncol. 2016;11(10):1774-84.

8. lasonos A, Schrag D, Raj GV, Panageas KS. How to build and interpret a nomogram for cancer prognosis. J Clin Oncol. 2008;26(8):1364-70.

9. Balachandran VP, Gonen M, Smith JJ, DeMatteo RP. Nomograms in oncology: more than meets the eye. Lancet Oncol. 2015;16(4):e173-80.

10. Semenkovich TR, Yan Y, Subramanian M, Meyers BF, Kozower BD, Nava R, et al. A Clinical Nomogram for Predicting Node-positive Disease in Esophageal Cancer. Ann Surg. 2019.

11. Tang X, Zhou X, Li Y, Tian X, Wang Y, Huang M, et al. A Novel Nomogram and Risk Classification System Predicting the Cancer-Specific Survival of Patients with Initially Diagnosed Metastatic Esophageal Cancer: A SEER-Based Study. Ann Surg Oncol. 2019;26(2):321-8.

12. Du F, Sun Z, Jia J, Yang Y, Yu J, Shi Y, et al. Development and Validation of an Individualized Nomogram for Predicting Survival in Patients with Esophageal Carcinoma after Resection. J Cancer. 2020;11(14):4023-9. 
13. Oki S, Toiyama Y, Okugawa Y, Shimura T, Okigami M, Yasuda H, et al. Clinical burden of preoperative albumin-globulin ratio in esophageal cancer patients. Am J Surg. 2017;214(5):891-8.

14. Yodying $H$, Matsuda A, Miyashita M, Matsumoto S, Sakurazawa N, Yamada M, et al. Prognostic Significance of Neutrophil-to-Lymphocyte Ratio and Platelet-to-Lymphocyte Ratio in Oncologic Outcomes of Esophageal Cancer: A Systematic Review and Meta-analysis. Ann Surg Oncol. 2016;23(2):646-54.

15. Okadome K, Baba Y, Yagi T, Kiyozumi Y, Ishimoto T, Iwatsuki M, et al. Prognostic Nutritional Index, Tumor-infiltrating Lymphocytes, and Prognosis in Patients with Esophageal Cancer. Ann Surg. 2020;271(4):693-700.

16. Chen S, Li X, Wen X, Peng S, Xue N, Xing S, et al. Prognostic nomogram integrated baseline serum lipids for patients with non-esophageal squamous cell carcinoma. Ann Transl Med. 2019;7(20):548.

17. Shephard DA. The 1975 Declaration of Helsinki and consent. Can Med Assoc J. 1976;115(12):11912.

18. Sun K, Chen S, Xu J, Li G, He Y. The prognostic significance of the prognostic nutritional index in cancer: a systematic review and meta-analysis. J Cancer Res Clin Oncol. 2014;140(9):1537-49.

19. Koziol JA, Jia Z. The concordance index $C$ and the Mann-Whitney parameter $\operatorname{Pr}(X>Y)$ with randomly censored data. Biom J. 2009;51(3):467-74.

20. Edge SB, Compton CC. The American Joint Committee on Cancer: the 7th edition of the AJCC cancer staging manual and the future of TNM. Ann Surg Oncol. 2010;17(6):1471-4.

21. Callegaro D, Miceli R, Bonvalot S, Ferguson P, Strauss DC, Levy A, et al. Development and external validation of two nomograms to predict overall survival and occurrence of distant metastases in adults after surgical resection of localised soft-tissue sarcomas of the extremities: a retrospective analysis. Lancet Oncol. 2016;17(5):671-80.

22. Liang W, Zhang L, Jiang G, Wang Q, Liu L, Liu D, et al. Development and validation of a nomogram for predicting survival in patients with resected non-small-cell lung cancer. J Clin Oncol. 2015;33(8):861-9.

23. Pulvirenti A, Javed AA, Landoni L, Jamieson NB, Chou JF, Miotto M, et al. Multi-institutional Development and External Validation of a Nomogram to Predict Recurrence After Curative Resection of Pancreatic Neuroendocrine Tumors. Ann Surg. 2019.

24. Chen S, Lai Y, He Z, Li J, He X, Shen R, et al. Establishment and validation of a predictive nomogram model for non-small cell lung cancer patients with chronic hepatitis B viral infection. J Transl Med. 2018;16(1):116.

25. Zhao Y, Li G, Zheng D, Jia M, Dai W, Sun Y, et al. The prognostic value of lymph node ratio and log odds of positive lymph nodes in patients with lung adenocarcinoma. J Thorac Cardiovasc Surg. 2017;153(3):702-9 e1.

26. Sakata J, Wakai T, Matsuda Y, Ohashi T, Hirose Y, Ichikawa H, et al. Comparison of Number Versus Ratio of Positive Lymph Nodes in the Assessment of Lymph Node Status in Extrahepatic 
27. Ferlay J, Colombet M, Soerjomataram I, Mathers C, Parkin DM, Pineros M, et al. Estimating the global cancer incidence and mortality in 2018: GLOBOCAN sources and methods. Int $\mathrm{J}$ Cancer. 2019;144(8):1941-53.

28. Gao RN, Neutel $\mathrm{Cl}$, Wai E. Gender differences in colorectal cancer incidence, mortality, hospitalizations and surgical procedures in Canada. J Public Health (Oxf). 2008;30(2):194-201.

29. Strasser B, Volaklis K, Fuchs D, Burtscher M. Role of Dietary Protein and Muscular Fitness on Longevity and Aging. Aging Dis. 2018;9(1):119-32.

30. Fournet M, Bonte F, Desmouliere A. Glycation Damage: A Possible Hub for Major Pathophysiological Disorders and Aging. Aging Dis. 2018;9(5):880-900.

31. Hipkiss AR. Aging, Alzheimer's Disease and Dysfunctional Glycolysis; Similar Effects of Too Much and Too Little. Aging Dis. 2019;10(6):1328-31.

32. Shao Y, Ning Z, Chen J, Geng Y, Gu W, Huang J, et al. Prognostic nomogram integrated systemic inflammation score for patients with esophageal squamous cell carcinoma undergoing radical esophagectomy. Sci Rep. 2015;5:18811.

33. Gasparyan AY, Ayvazyan L, Mukanova U, Yessirkepov M, Kitas GD. The Platelet-to-Lymphocyte Ratio as an Inflammatory Marker in Rheumatic Diseases. Ann Lab Med. 2019;39(4):345-57.

34. Afari ME, Bhat T. Neutrophil to lymphocyte ratio (NLR) and cardiovascular diseases: an update. Expert Rev Cardiovasc Ther. 2016;14(5):573-7.

35. Roxburgh CS, McMillan DC. Role of systemic inflammatory response in predicting survival in patients with primary operable cancer. Future Oncol. 2010;6(1):149-63.

36. Suh B, Park S, Shin DW, Yun JM, Keam B, Yang HK, et al. Low albumin-to-globulin ratio associated with cancer incidence and mortality in generally healthy adults. Ann Oncol. 2014;25(11):2260-6.

37. Gabay C, Kushner I. Acute-phase proteins and other systemic responses to inflammation. N Engl J Med. 1999;340(6):448-54.

38. Vavricka SR, Burri E, Beglinger C, Degen L, Manz M. Serum protein electrophoresis: an underused but very useful test. Digestion. 2009;79(4):203-10.

39. Wu PC, Posner MC. The role of surgery in the management of oesophageal cancer. Lancet Oncol. 2003;4(8):481-8.

40. Visser E, Markar SR, Ruurda JP, Hanna GB, van Hillegersberg R. Prognostic Value of Lymph Node Yield on Overall Survival in Esophageal Cancer Patients: A Systematic Review and Meta-analysis. Ann Surg. 2019;269(2):261-8.

41. Tan Z, Ma G, Yang H, Zhang L, Rong T, Lin P. Can lymph node ratio replace pn categories in the tumor-node-metastasis classification system for esophageal cancer? J Thorac Oncol. 2014;9(8):1214-21.

42. Mariette C, Piessen G, Briez N, Triboulet JP. The number of metastatic lymph nodes and the ratio between metastatic and examined lymph nodes are independent prognostic factors in esophageal 
cancer regardless of neoadjuvant chemoradiation or lymphadenectomy extent. Ann Surg. 2008;247(2):365-71.

43. Cao J, Yuan P, Ma H, Ye P, Wang Y, Yuan X, et al. Log Odds of Positive Lymph Nodes Predicts Survival in Patients After Resection for Esophageal Cancer. Ann Thorac Surg. 2016;102(2):424-32.

44. Obuchowski NA, Bullen JA. Receiver operating characteristic (ROC) curves: review of methods with applications in diagnostic medicine. Phys Med Biol. 2018;63(7):07TR1.

\section{Figures}

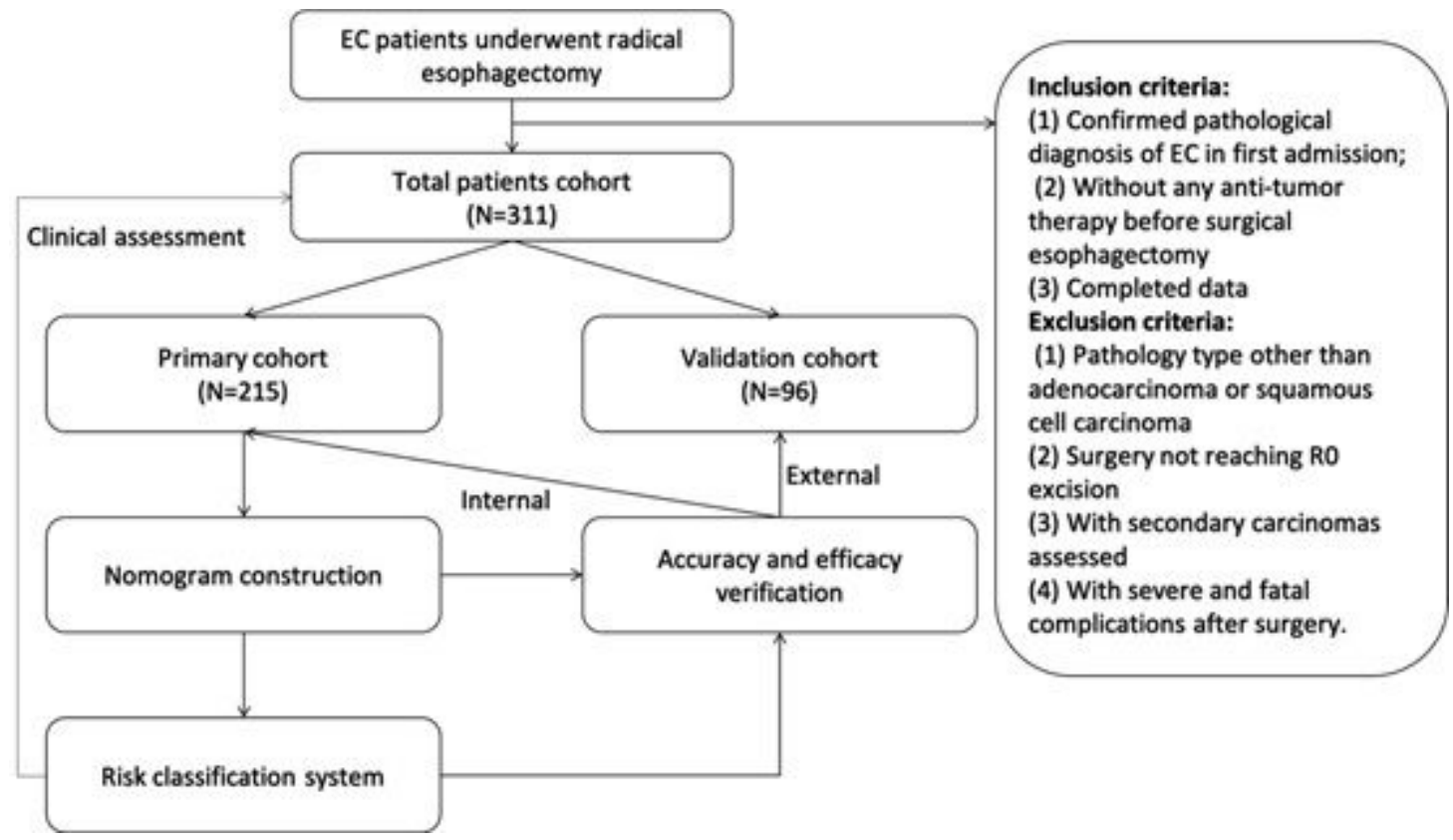

\section{Figure 1}

A flow chart for the design of our study. A total of $311 \mathrm{EC}$ patients underwent radical esophagectomy were randomly divided into primary and validation cohorts. A nomogram was built based on the primary cohort, the accuracy and efficacy of the nomogram and relevant risk classification system were verified in both primary and validation cohort. Finally, the clinical assessment of risk classification system was performed in total patients. EC: Esophageal cancer. 
Points

0

12

23

34

4

6

7

8

9 10

age

gender

AGR

N

PRL

PNI

Total Points

1-year OS

3-year OS

5-year OS
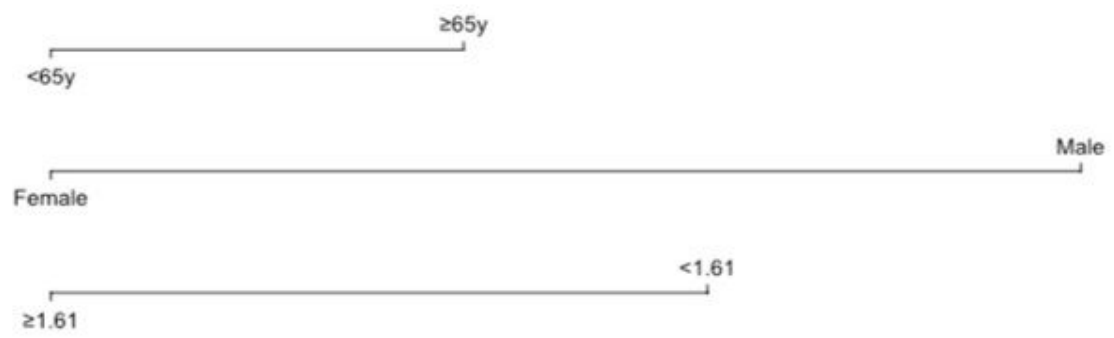

N2-N3

$$
\text { No-N1 }
$$

$\geq 0.1$

$$
<0.1
$$
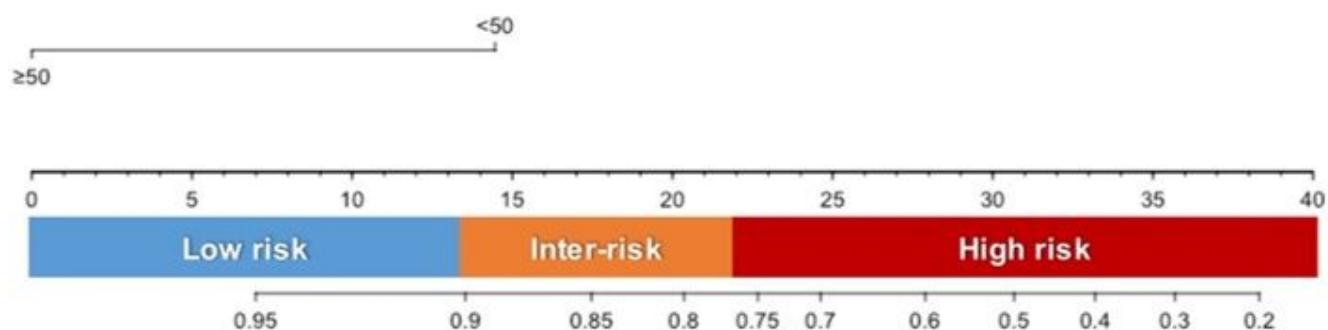

$\begin{array}{llll}0.85 & 0.8 & 0.75 & 0.7\end{array}$

$\begin{array}{lllll}1 & 1 & 1 & 1 & \\ 0.5 & 0.5 & 0.4 & 0.3 & 0.2\end{array}$
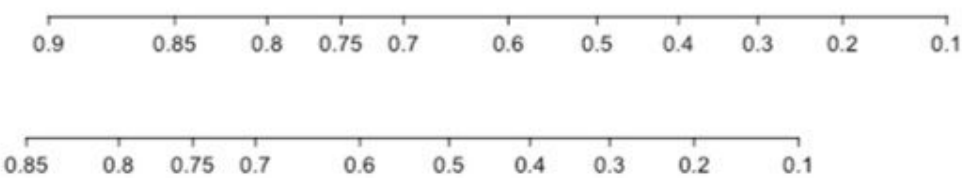

\section{Figure 2}

Nomogram prediction model for OS of EC patients underwent radical esophagectomy. As showed above, six variables correspond to the upper points scale respectively, while the sum score of each variable reaches downward to the total points which is related to the prediction of 1-, 3-, 5-year overall survival rate. Moreover, a line bar composed of three colors indicates three risk group according to the predictive OS. Furthermore, the risk status of radical esophagectomy EC patients would be obtained based on the nomogram. AGR: albumin to globulin ratio, PRL: positive lymph nodes ratio, PNI: Prognostic Nutritional Index, N: modified N staging, Inter-risk: Intermediate risk. 

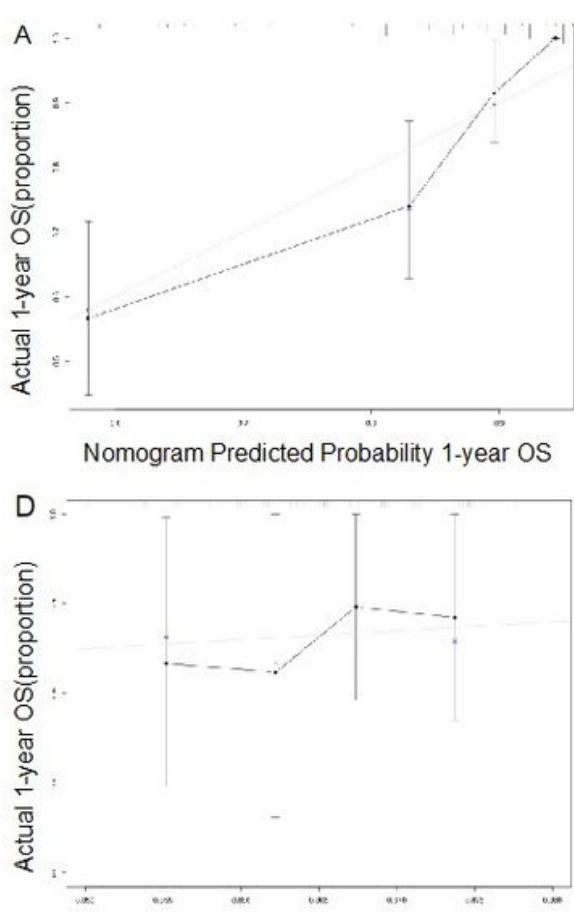

Nomogram Predicted Probability 1-year OS
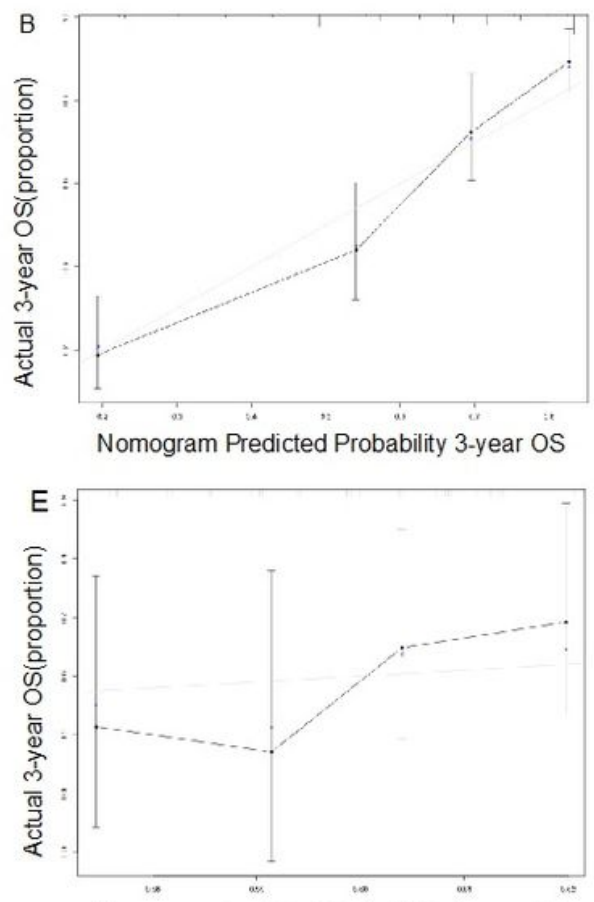

Nomogram Predicted Probability 3-year OS
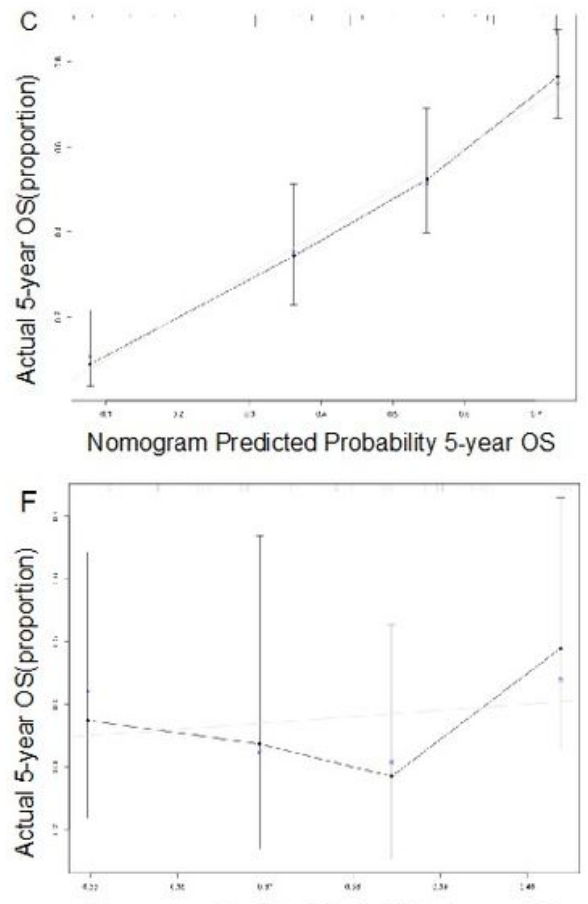

Nomogram Predicted Probability 5-year OS

\section{Figure 3}

Calibration curves of nomogram model in predicting OS of EC patients. 1-, 3-, 5-year OS probability and actual 1-, 3-, 5-year OS in primary cohort (A-C). Calibration curves of nomogram model predicting 1-, 3-, 5year OS probability and actual 1-, 3-, 5-year OS in validation cohort (D-F). The closer curve is to the calibration dotted line, the more accurate the prediction capacity of nomogram. OS: Overall survival.
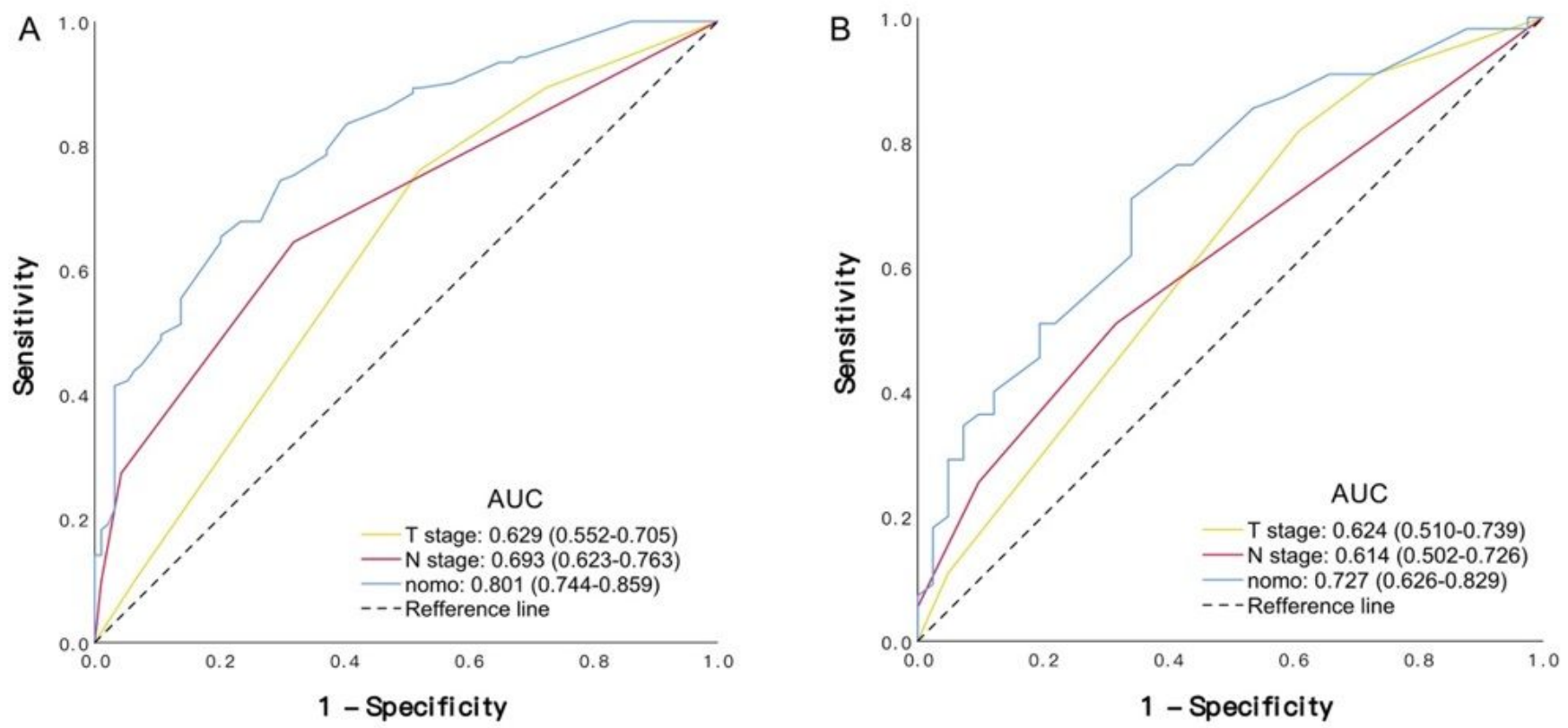

\section{Figure 4}


ROC curves of TNS calculated from nomogram model for the OS prediction were performed in both primary cohort (A) and validation cohort (B) compared with 7th AJCC $T$ and $N$ stage. The larger value of AUC reveals a better capability for OS prediction, ROC: receiver operating characteristics, TNS: total nomogram-related score, AUC: area under curve, nomo: Nomogram.
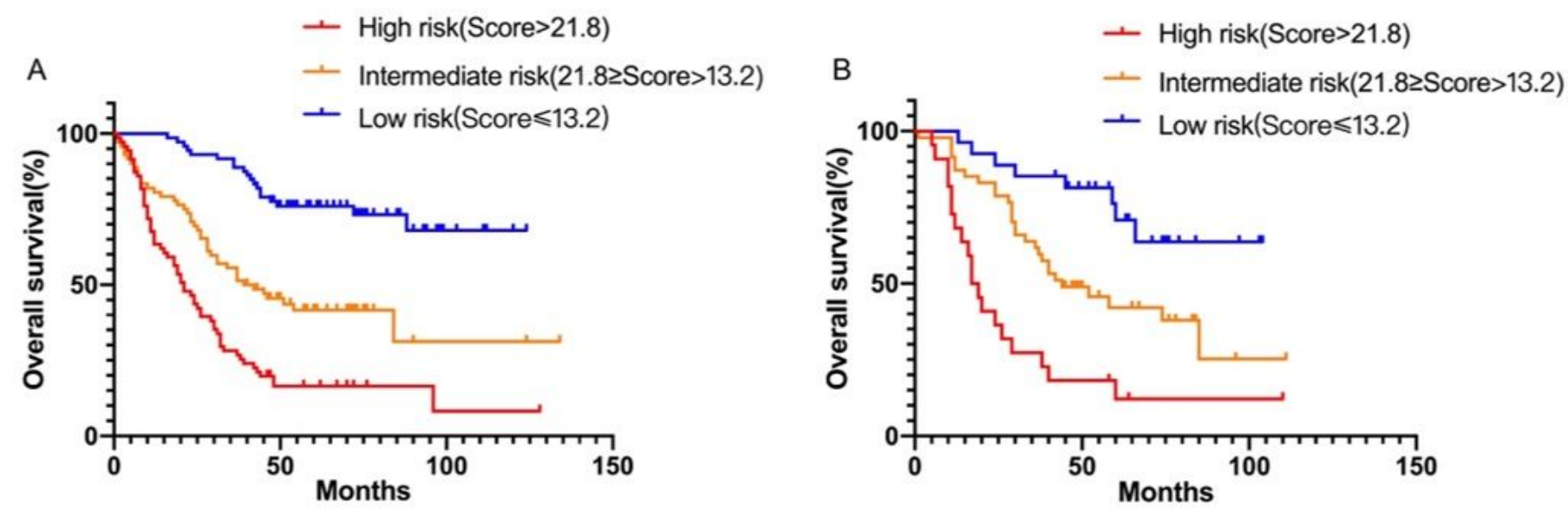

Figure 5

Kaplan-Meier curves for the OS of EC patients in the low, intermediate and high risk groups of primary (A) and validation cohorts (B), respectively. The cut-off scores were determined by TNS in primary cohort. OS: Overall survival, TNS: total nomogram-related score.
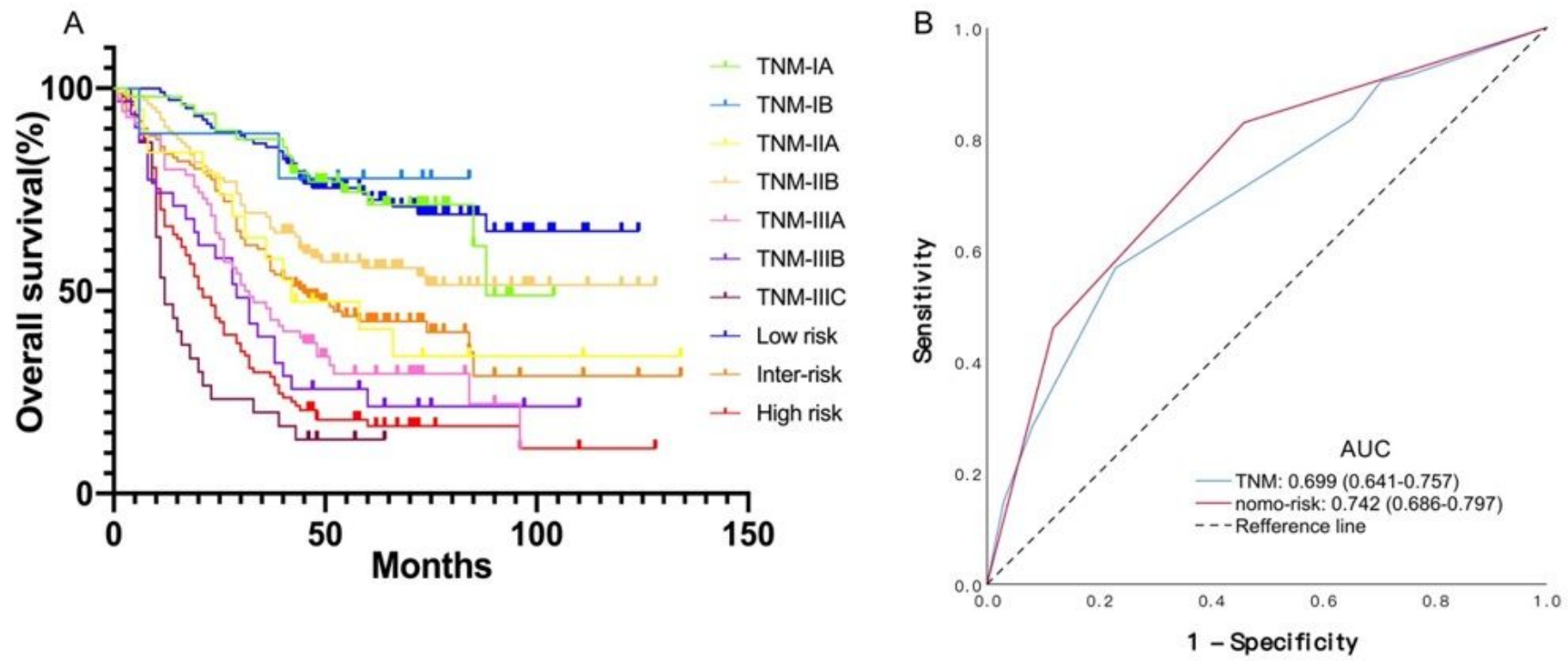

Figure 6

Kaplan-Meier curves for the OS of EC patients in AJCC TNM stages and nomogram-related risk groups (A), ROC curves of AJCC TNM stages and nomogram-related risk groups in predicting OS in EC patients Loading [MathJax]/jax/output/CommonHTML/jax.js am-related risk classification system, Inter-: Intermediate, 
AJCC TNM: tumor-node-metastasis staging system represented by 7th American Joint Committee on Cancer. 